\title{
II passato, il presente e il futuro degli strumenti di misura delle variabili idrogeologiche
}

\section{Francesco La Vigna}

ROMA CAPITALE Dip. Tutela ambientale - Protezione Civile

Uff. Geologia ed Idrogeologia Ambientale - Roma

francesco.lavigna@comune.roma.it

Contrariamente al geologo, l'idrogeologo raramente si serve di bussola, lente e martello, perchè dovendo intercettare una realtà fisica per natura quasi sempre "nascosta" deve, ahimè, sempre sovraccaricarsi dei propri particolari strumenti del mestiere che nella più leggera delle ipotesi sono costituiti almeno da una piuttosto pesante bobina metrata di filo elettrico, alimentata a batteria, e fornita di cicalino (ossia il freatimetro). Sebbene le misure che un idrogeologo di campo svolge nei suoi rilevamenti non siano cambiate dalla nascita di questa disciplina, l'innovazione tecnologica ha fatto e continua a fare notevoli passi avanti mettendo a disposizione strumenti sempre più precisi, rapidi ed interfacciabili con applicativi software.

Relativamente alle misure in foro e al classico freatimetro, la cui tecnologia di base ha resistito nel tempo, si segnala la possibilità che alcuni nuovi prodotti permettono di equipaggiare le sonde con rilevatori di diverso tipo, come termometri e conduttimetri, senza aumentare il diametro della sonda, permettendo quindi di effettuare dei $\log$ verticali chimico-fisici, altrimenti effettuabili con ingombranti sonde multi-parametriche. Inoltre alcuni freatimetri di ultima generazione possono anche essere equipaggiati con sensori di fondo foro (Fig.1).

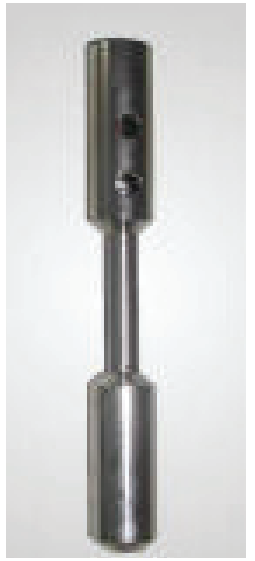

Fig. 1 - Punta sensore di fondo foro per freatimetri (foto da bttp:l/freatimetri.geoexpo.itffreatimetri.htm).

Restando alle misure in foro, se gli ultimi decenni sono stati caratterizzati dallo sviluppo sempre più sofisticato di sensori per il rilevamento in continuo di livello, temperatura e conducibilità elettrica, facendo si che oggi ne esistano di svariate tipologie, con dimensioni minime, con grande autonomia di carica e a prezzi un tempo inimmaginabili, le tendenze più recenti della ricerca sono quelle dedite allo sviluppo di strumenti in grado di fornire informazioni circa i flussi di falda attraverso i filtri di pozzi o piezometri. La maggior parte di questi strumenti usa la genesi di un campo di calore e restituisce le direzioni di flusso prevalenti sulla base di come questo campo sia modificato nel tempo. Se però qualche anno fa la strumen- tazione di questo tipo era piuttosto ingombrante e pesante, oggi si fanno strada le fibre ottiche e la tecnologia DTS (Distributed Temperature Sensing), tecniche leggere, meno ingombranti e in grado di fornire una risoluzione maggiore (Culshaw 2005; Selker 2008; Tyler et al. 2008). Uno strumento che ritengo però davvero innovativo e che facilita e snellisce drasticamente i tempi di rilevamento piezometrico è il cosiddetto "sonic water table meter" (Fig.2) ossia un misuratore di livello piezometrico ad impulsi sonori. Questo strumento permette, tramite l'emissione di impulsi sonori, di determinare con ottima approssimazione la profondità del livello di falda senza dover calare alcuna sonda all'interno del foro, evitando quindi, soprattutto nei pozzi attrezzati di rischiare che il terminale del freatimetro si vada ad incastrare con la pompa, con i cavi ad essa connessa o con eventuali centratori. La misura è molto rapida e con un buon grado di approssimazione (+/- 0,2\%), purché si conosca il range di profondità da indagare (normal 0-60 m o deep 40$350 \mathrm{~m}$ ) mentre per la misura occorre semplicemente che esista almeno un piccolo foro attraverso cui far passare gli impulsi sonori.

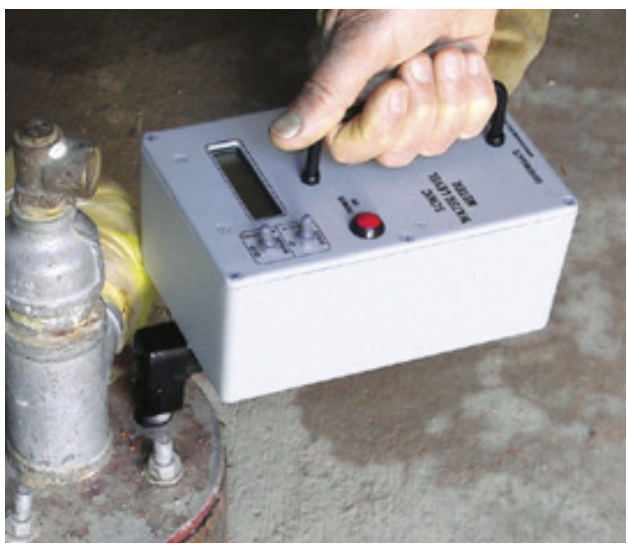

Fig. 2 - Misuratore di livello freatimetrico ad impulsi sonori "Sonic water tebale mater" (foto da www.envirotechonline.com).

Sempre relativamente alla dimensione verticale sono invece da segnalare delle nuove tecnologie che da diverso tempo sono disponibili in campo ambientale per effettuare campionamenti multilivello a partire dallo stesso foro. Esistono infatti ormai dei tubi piezometrici che possono indagare fino a 7 livelli diversi lungo la stessa verticale (Fig.3 CMT - Continous Multichannel Tubing) aiutando quindi a ricostruire con migliore accuratezza, ad esempio, su quale orizzonte siano concentrati eventuali agenti contaminanti (Stansbery et al. 2001). Sempre riguardo a questi aspetti è da segnalare la tecnologia "Direct Push" (Fig.4) che prevede, tramite l'utilizzo di un penetrometro 
statico, l'infissione di aste cave dotate di un filtro apribile alla profondità desiderata attraverso cui, quindi, poter effettuare dei campionamenti mirati senza dover eseguire perforazioni (Dietrich e Leven 2006). Tramite la stessa tecnica è inoltre possibile parametrizzare gli acquiferi attraverso dei log di resistività (Vienken et al. 2014)

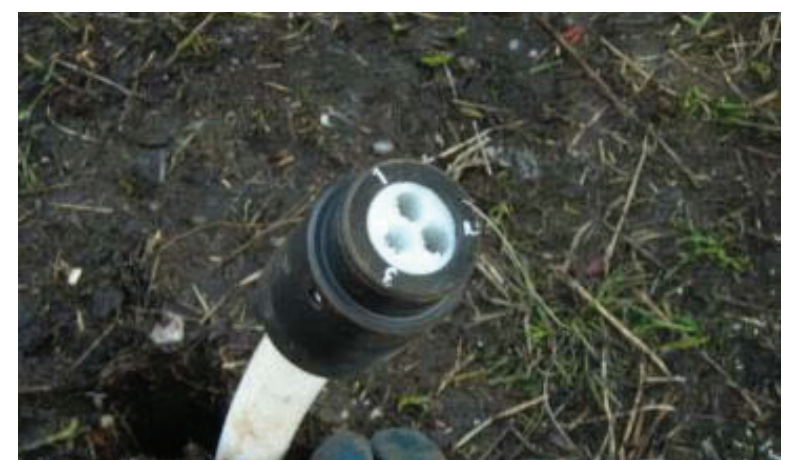

Fig. 3 - Tubo piezometrico multiplo per campionamenti a diverse profondità (CMT - Coninous Multichannel Tubing) (Foto da http://www.solinst.com).

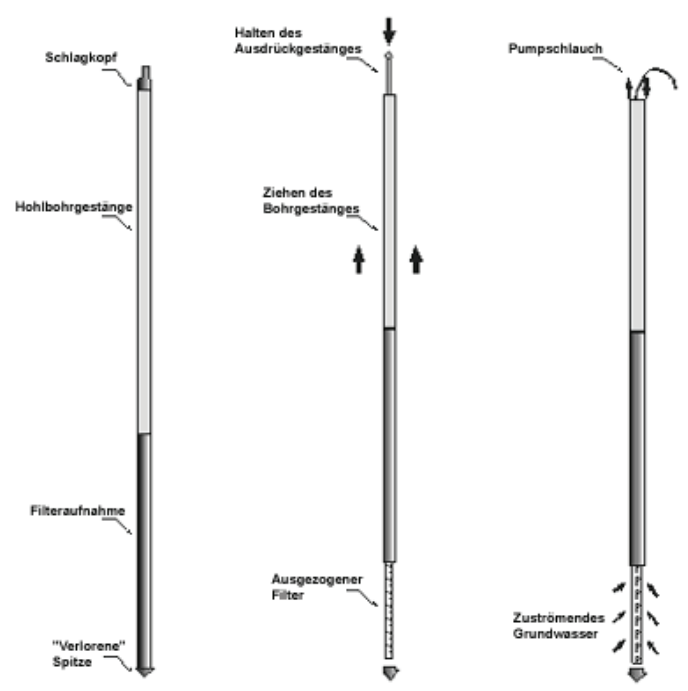

Fig. 4 - Schema di funzionamento della tecnologia "direct push" (Foto da http://www.tueg-umwelt.com).

Relativamente alle misure di portata in alveo già da diversi anni sono state realizzate nuove tipologie di sensori con l'intento di superare il mulinello idrometrico meccanico o correntometro ad elica, che però continua ad essere usato correntemente. Sono infatti in commercio da molto tempo dei misuratori che sfruttano lo stesso concetto del mulinello sfruttando altre tecnologie come l'induzione magnetica o gli impulsi sonori. Questi strumenti hanno raggiunto un notevole grado di accuratezza, permettendo di poter determinare anche velocità di flusso inferiori al cm/s. Nell'ultimo decennio, i misuratori ad impulsi sonori sono stati poi montati su natanti dotati di GPS che tramite l'utilizzo di software evoluti sono in grado di ricostruire il solido di portata di una sezione di misura molto rapidamente e con buon grado di approssimazione, semplicemente "traghettando" il natante da una parte all'altra del corso d'acqua (Fig.5). Sebbene molto evolute, anche queste tecniche di misura della portata dei corsi d'acqua sono destinate ad essere superate da nuove tecnologie emergenti. Sono infatti già in uso in via sperimentale le fibre ottiche per determinare con precisione tratti di alveo drenanti o disperdenti sempre per mezzo della tecnologia DTS (Distributed Temperature Sensing) che si basa sulla misura dellamodificazione del campo di temperatura generato (Lane et al. 2008; Selker et al. 2006).

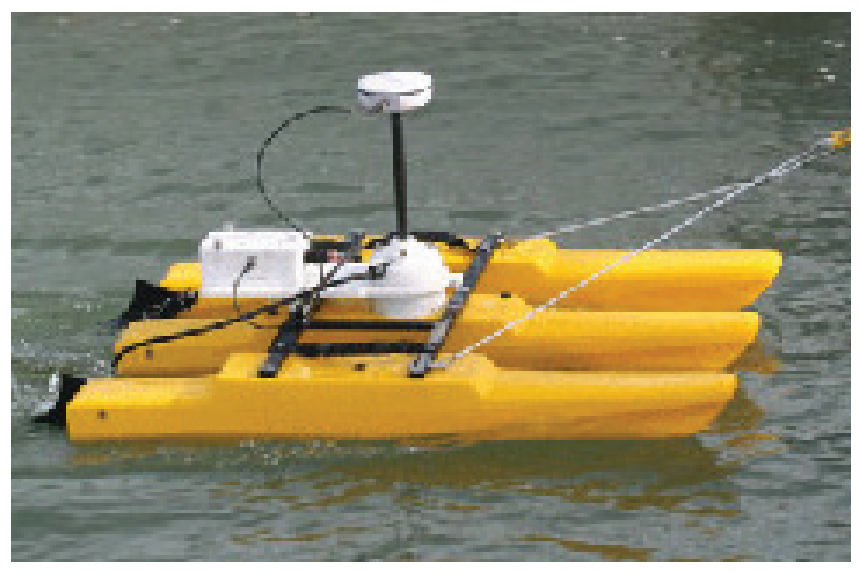

Fig. 5 - Natante per la misura della portata dei corsi d'acqua (Foto da bttp:// www. sontek.com).

\section{BIBLIOGRAFIA}

Culshaw B (2005). Fiber-Optic Sensors: Applications and Advances. The International Conference on Optical Fiber Sensors OFS17 - Belgium

Dietrich P, Leven C (2006). Direct Push-Technologies. In Kirsch R (Ed) Groundwater Geophysics. SpringerDoi:10.1007/3-540-29387-6_11

Lane JW, Day-Lewis FD, Johnson CD, Dawson CB, Nelms DL, Eddy-Miller CA, Wheeler JD, Harvey CF, Karam H (2008). Fiber-optic distributed temperature sensing: A new tool for assessment and monitoring of hydrologic processes. Symposium on the Application of Geophysics to Engineering and Environmental Problems, April 6-10, 2008, Philadelphia, Pennsylvania, Proceedings: Denver, Colorado, Environmental and Engineering Geophysical Society, 9 p.

Selker J (2008). Temperature Fiber Optics For Environmental Sensing. Sensors

Selker J, Van de Giesen N, Westhoff M, Luxemburg W, Parlange MB (2006). Fiber optics opens window on stream dynamics. Geophysical Research Letters, 33 :24, DOI: 10.1029/2006GL027979

Tyler SW, Selker JS, Hausner MB, Hatch CE, Torgersen T, Thodal CE, Schladow SG (2008). Environmental temperature sensing using Raman spectra DTS fiber-optic methods. Water Resources Research, 45: W00d23, Doi:10.1029/2008wr007052

Stansbery A, Sandberg D, Truitt R (2001). Multi-Level Groundwater Monitoring System at Hunters Point Shipyard - A Demonstration of the Continuous Multichannel Tubing System. TechData Sheet Naval Facilities Engineering Service Center Port Hueneme, CA, TDS-2073-ENV

Vienken T, Kreck M, Hausmann J, Werban U, Dietrich P (2014). Innovative strategies for high resolution site characterization: applicationto a flood plain. Acque Sotterranee - Italian Journal of Groundwater, 4:138 DOI 10.7343/AS-091-14-0118 